\title{
A simple scheme for documenting corneal disease
}

\author{
A. J. BRON \\ Institute of Ophthalmology, University of London
}

Although a generally accepted convention exists for the graphic documentation of retinal disorders (Schepens, I969), no similarly acceptable scheme is available for the documentation of corneal diseases. This diminishes the value of many clinical records since each observer uses a personal method of recording his observations which, although possibly standardized for himself, is not meaningful to other clinicians.

With this in mind, the author has devised a simple notation for use in drawing diseased corneae which has been in use for the past 2 years. It is presented here as a scheme which other clinicians may find of value.

\section{Methods}

THE CORNEA

Plan View

The cornea is depicted in plan view by a circle which represents the corneal as opposed to the scleral limbus. Vessels drawn inside the circle are therefore by definition pathological. If it is required to depict the vessels of the limbal palisades, these must be drawn outside the circle, as must other limbal features such as limbal infiltrates and follicles, etc. The pupil is shown, if required, by a dashed line (Fig. I $a$ ). Different letter symbols are used to represent the different features of corneal disease. Fig. $\mathrm{I} a-j$ shows how the symbols may be employed in various clinical situations, and their detailed use is outlined below.

In order to indicate the level of a particular feature on the plan diagram, additional annotation is necessary to state whether the feature is in the epithelium, stroma, Descemet's membrane, or endothelium. The following abbreviations are used:

Ep. = Epithelium

Str. = Stroma

Des. = Descemet's membrane

End. = Endothelium

The location of a particular change in the stroma may be further identified by indicating whether the feature is present in the anterior, middle, or posterior stroma. The following abbreviations are used:

$$
\mathrm{a}=\text { anterior; } \mathrm{m}=\text { middle; } \quad \mathrm{p}=\text { posterior }
$$

These letters are written as a subscript to the symbol letter for the feature observed, e.g. a midstromal infiltrate would be annotated:

Str. $I_{m}-$ or just $I_{m}$ - for short 
$(a)$

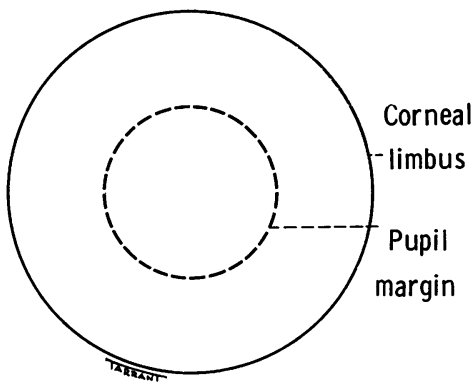

$(c)$
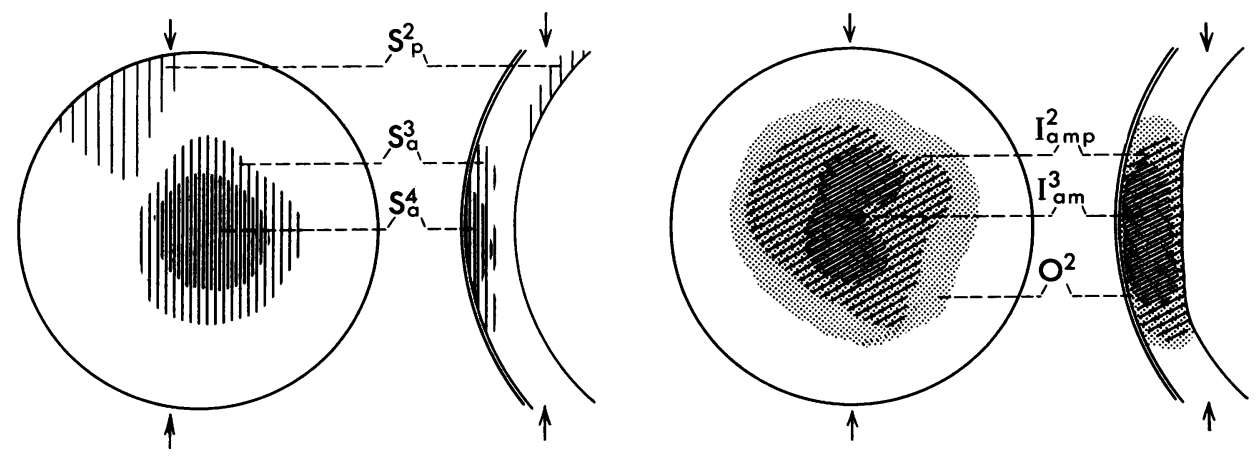

(e)
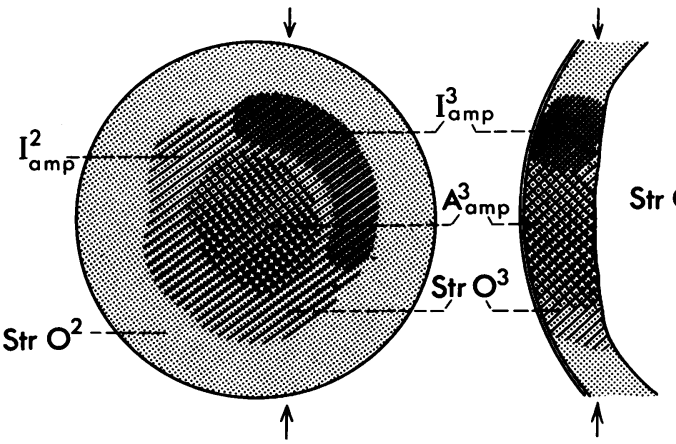

FIG. Ia Plan view of cornea

FIG. Ib Corneal oedema due to endothelial dysfunction (e.g. Fuchs's dystrophy).

FIG. IC Stromal corneal scars of differing density located at different stromal levels

FI G. Id Axial stromal infiltration and oedema

FIG. Ie Axial stromal abscess surrounded by infiltrate at different levels. Stromal oedema also present

FIG. If Corneal oedema with a zone of scarring and irregular thinning (Thickness shown by the prefix " $T$ " followed by the decimal fraction of normal thickness)

The degree of change is denoted by a grading system on a $I-4$ scale:

$$
\begin{array}{ll}
1=\text { minimal change } & 3=\text { moderate change } \\
2=\text { mild change } & 4=\text { severe change }
\end{array}
$$

and this is recorded as a superscript after the symbol letter, e.g. $\mathrm{I}^{2}=$ an infiltrate of mild degree, or $\mathbf{I}_{\mathbf{a}}{ }^{2}=$ an anterior stromal infiltrate of mild degree.

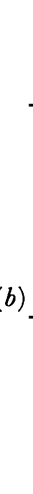

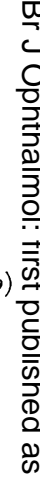

$\overrightarrow{0}$

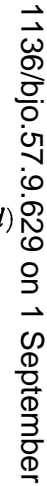



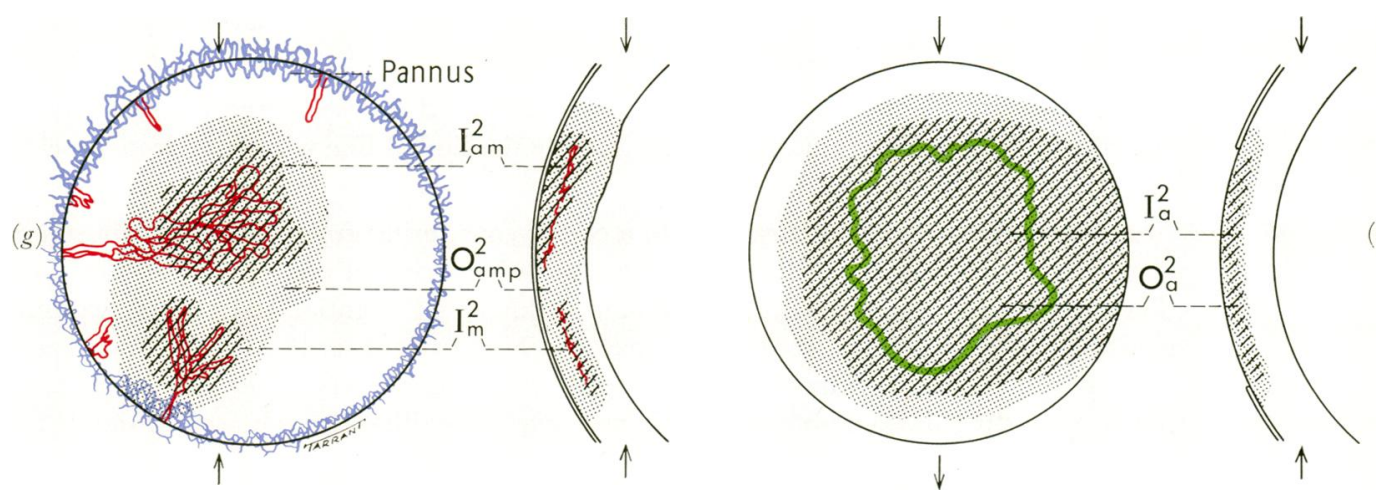

(i)
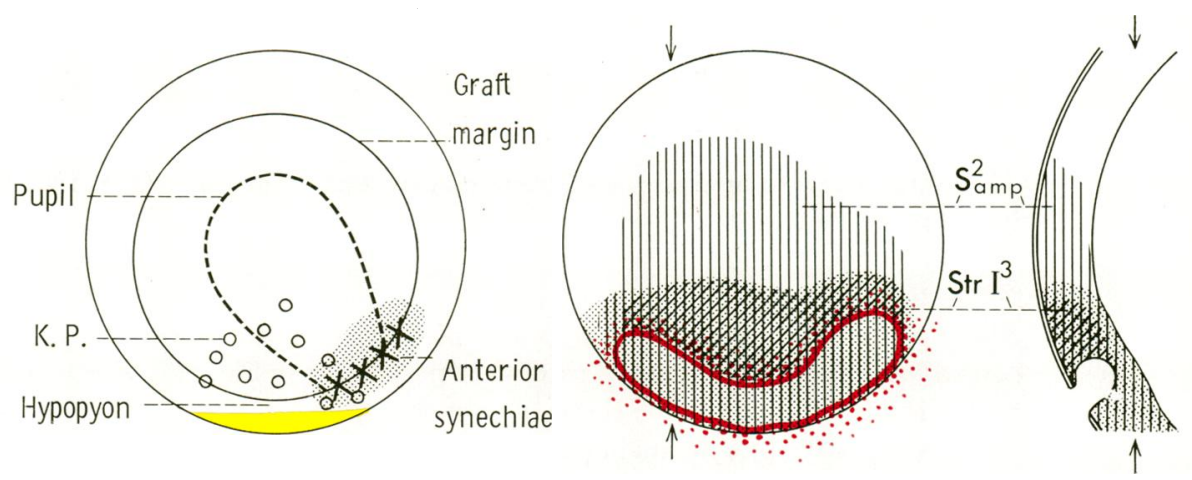

FIG. Ig Corneal infiltration and oedema with deep and superficial vascularization

FIG. I $h$ Anterior stromal infiltration and oedema with an overlying erosion as stained with fluorescein

FIG. I $i$ Corneal graft with corneal oedema related to the site of anterior synechiae at the graft/host junction. The pupil is deformed and elongated, there are keratic precipitates over the lower portion of the graft, and a hypopyon is present

FIG. I $j$ Cornea showing extensive stromal scarring and an elongated marginal ulcer below. The ulcer is undermined on one side and shelved on the other. It has been stained with bengal rose. There is an infiltrate at the axial margin of the ulcer

The following symbols are employed to describe individual features:

Corneal oedema is represented by pencil shading and is annotated with the letter " $\mathrm{O}$ " for oedema (Fig. $\mathrm{I} b$ ).

Corneal scarring or opacity is represented by vertical hatching and is annotated with the letter " $\mathrm{S}$ " for scar (Fig. IC).

Corneal infiltrate is represented by oblique single hatching annotated with the letter " $I$ "

Corneal abscess is represented by oblique double hatching annotated with the letter "A"

(Fig. I $d$ ).

Better definition may be achieved by outlining individual features.

(Fig. I $e$ ).

(As stated above, the degree of change is denoted by a grading system on a $\mathrm{r}$ to 4 scale. In addition, it may be represented on the drawing by varying the density of shading or hatching; closer hatching or denser shading signifying a greater degree of change (Fig. I $b-e)$ ).

Corneal thickness may be indicated on the plan view of the cornea by expressing the estimated thickness at any given point as a decimal fraction of normal corneal thickness. The drawing is annotated with the letter " $T$ " for thickness, followed by the decimal number (Fig. I $f$ ). Where 
a cornea shows marked thickness variation at multiple sites, such a course may be preferable to the drawing of multiple sectional views.

Corneal vessels are depicted using a colour code; red for deep vessels and blue for superficial vessels. The superficial vessels are drawn starting outside the limbal line while the deep vessels arise at the limbal line (Fig. $\mathrm{I} g$ ).

Staining reactions are also indicated with a colour code, using green for fluorescein staining and red for staining with bengal rose. An ulcer is shown by a ring of appropriate shape in red or green. (The area may be shaded with the colour but this is not essential and indeed may obscure other details which have been entered on the chart. The use of red for both deep vessels and bengal rose staining is not ambiguous in use since the features shown are very different.) Punctate staining is shown by dotting in the appropriate colour (Fig. $\mathrm{I} h$ ). Blue may be used to signify staining with Alcian blue.

\section{KERATIC PRECIPITATES}

These are indicated as small round circles annotated with the letters "K.P." (Fig. I $i$ ).

\section{ANTERIOR SYNEGHIAE}

These are indicated as crosses and annotated by writing in full (Fig. $\mathrm{I} i$ ). Hypopyon is indicated in yellow and annotated in writing (Fig. $\mathrm{I} i$ ).

SECTIONAL VIEWS

Although the above scheme attempts to depict many features of corneal disease on a plan view of the cornea, sectional views should be used when possible, as in Fig. I $b, c, d, e, g$, particularly where corneal profile is important, as in the shape of an ulcer (Fig. $\mathrm{I} j$ ).

The scheme enables the main features of corneal diseases to be shown, but there are many specific lesions which will require to be indicated in addition. Such features as Salzman's nodules, epithelial microcysts, lipid deposits, cornea guttata, and so on, would require additional annotation. A descriptive nomenclature for punctate keratitis has already been devised by Professor Barrie Jones and is in current use (i.e. Punctate Epithelial Erosion (P.E.E.), Punctate Epithelial Keratitis (P.E.K.), Combined Epithelial and Subepithelial Keratitis (C.E.K.), Subepithelial Keratitis (S.E.K.) : Jones 1960).

A further and most valuable annotation which should be made is an indication of the size and meridional location of certain lesions such as corneal ulcers.

Fig. 2 (opposite) is a reference chart for use in the clinic which allows clinicians unfamiliar with the scheme to make use of the recording system.

\section{Discussion}

Any attempt to formulize the recording of clinical observations is likely to focus attention on certain problems encountered in translating these observations into documentary terms. In corneal diseases, these problems relate mainly to the difficulty in identifying as distinct entities the features which have to be recorded in the clinical situation. For instance, the transition from "severe infiltrate" to "abscess" is difficult to identify. Also, the concept of a minimal or mild grade of abscess is not a valuable one and it is likely that clinical assessment of the grade of severity of an abscess is based as much on the general reaction of the globe and anterior segment as on the appearance of the cornea itself. For this reason, most clinicians will probably grade abscesses as moderately severe or severe (i.e. Grade 3 or 4$)$.

Another difficulty arising from the terminology relates to the use of the term "scar or opacity" as an entity distinct from oedema, infiltrate, or abscess. Each of the latter features contributes a certain amount of opacity to the cornea. Moreover, corneal oedema 


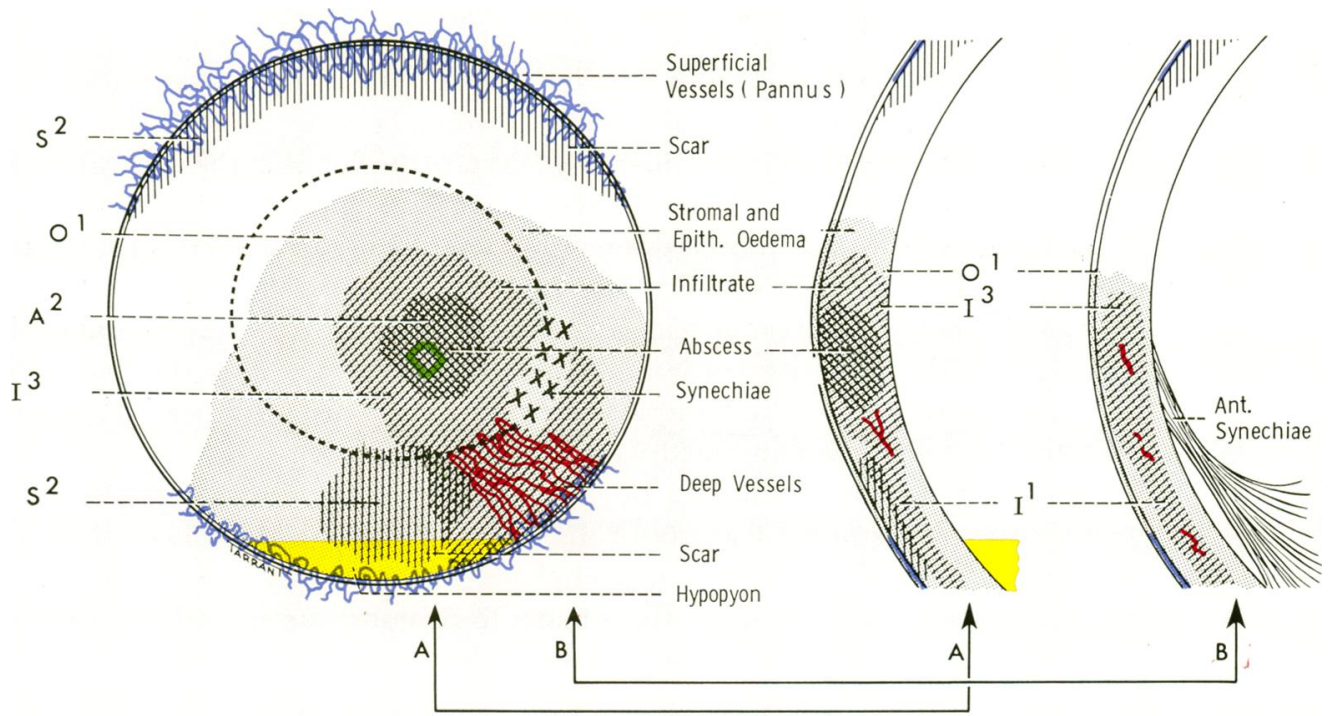

(Indicate position of sectional view :- A, B, C etc.)

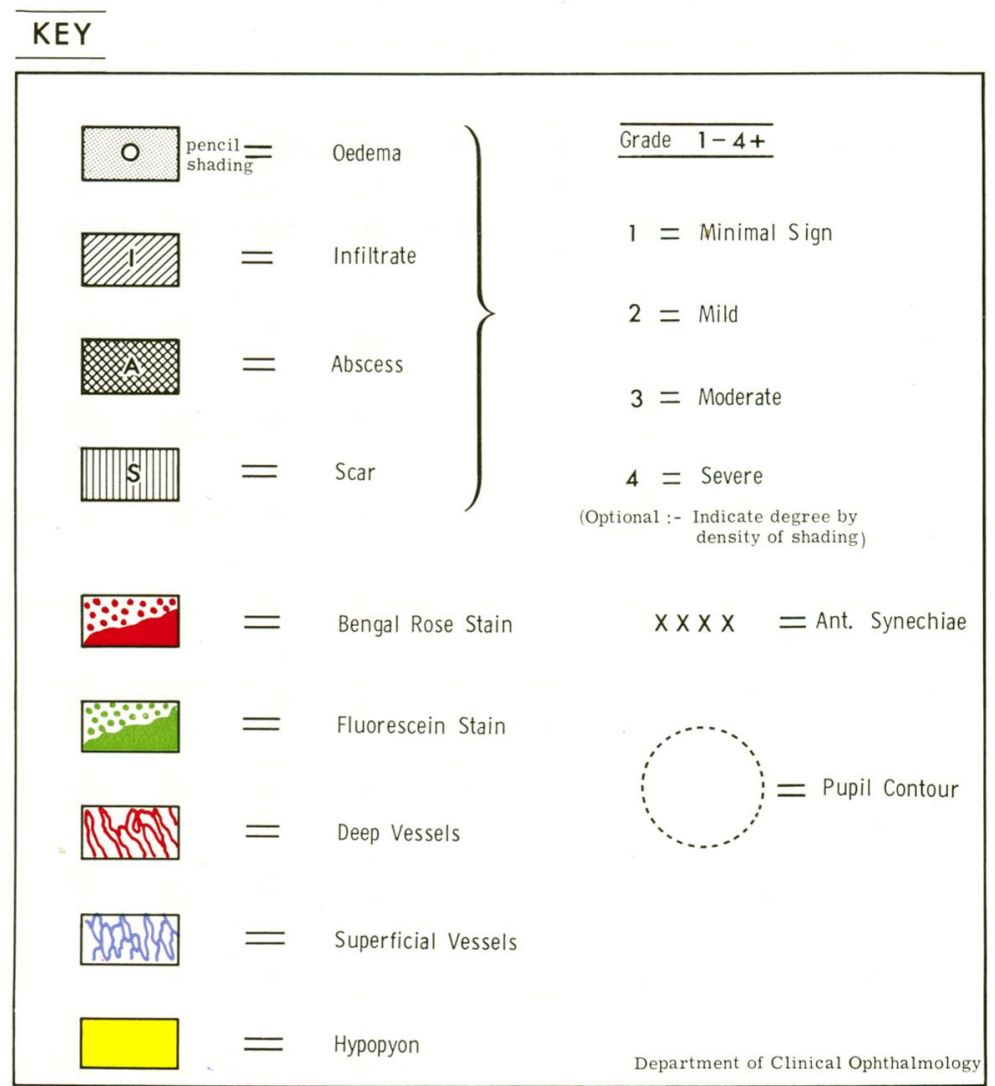

FIG. 2 Reference chart and key for documenting corneal disease, for use in the clinic (The statement " $O=$ Oedema" may be pasted over with $E=E$ Edema by American users) 
may obscure the presence of a fine infiltrate, and oedema or infiltrate may obscure the presence of opacity due to prior scarring. When a corneal inflammation is resolving and the oedema and infiltrate subsiding, the scar may become visible for the first time and will be recorded on the chart for the first time, despite the fact that the general level of "opacity" is diminishing. Also a chronic abscess or infiltrate may with improvement accumulate scar tissue, but this transition is difficult to identify and hence to record, because the degree of opacity does not alter significantly.

Again, when an abscess or scar is very large and dense, it may be impossible to identify the state of the posterior cornea. In this situation, it is important to indicate this uncertainty on the chart (the posterior corneal surface may be indicated by a dotted line, for instance, and may be annotated with a question mark).

Another problem which may be encountered is the failure of a schematic diagram to convey the pictographic features of the lesion drawn, i.e. the schematic drawing may fail to conjure up an image of the corneal changes in the mind of the observer. In fact, it is perfectly possible to over-draw the schema to demonstrate special features such as corneal folds, irregularities of the epithelium, and other unusual aspects of a corneal disorder.

My thanks are due to Prof. B. R. Jones for his interest during the preparation of this schema, and to Mr. T. R. Tarrant of the Audio-Visual Department of the Institute of Opthalmology, London, for producing the excellent line drawings.

\section{References}

JONES, B. R. (ig6o) Trans. ophthal. Soc. U.K., 80, 665

SGHEPENS C. L. (1969) 'Techniques of examination of the fundus periphery', in "Symposium on Retina and Retinal Surgery", Transactions of the New Orleans Academy of Ophthalmology, p. 39: Mosby, St. Louis 